\title{
Quantitative Chemical Mapping of Anisotropic Molecular Distributions on Gold Nanorods
}

Blanka E. Janicek ${ }^{1}$, Joshua G. Hinman ${ }^{2}$, Jordan H. Hinman ${ }^{2}$, Sang hyun Bae ${ }^{1}$, Meng Wu ${ }^{2}$, Huei-Huei Chang $^{2}$, Kenneth S. Suslick ${ }^{2}$, Catherine J. Murphy ${ }^{2}$, and Pinshane Y. Huang ${ }^{*}$

1. Department of Materials Science and Engineering, University of Illinois at Urbana-Champaign, Urbana, IL, USA 61801

2. Department of Chemistry, University of Illinois at Urbana-Champaign, Urbana, IL, USA 61801

* Corresponding author: pyhuang@illinois.edu

The ability to characterize and quantify anisotropic distributions of organic molecules at nanoparticle interfaces is a longstanding challenge in the realization and design of functional coatings, monodisperse synthesis, and colloidal assembly [1]. Here, we show that by using electron energy loss spectroscopy (EELS) spectral imaging on graphene substrates in an aberration-corrected scanning transmission electron microscope (STEM), we can directly visualize and quantify molecular distributions on gold nanorods (AuNRs). By contrasting the average distributions of two organic ligands across dozens of nanoparticles, we find that (16-mercaptohexadecyl)trimethylammonium bromide (MTAB) forms a uniform coating, while the distribution of cetyltrimethylammonium bromide (CTAB) his highly anisotropic. On average, CTAB binding density is reduced on the ends of gold nanorods, consistent with the higher reactivity of the nanorod ends reported in the literature [2]. Our results demonstrate the potential of our methods to directly probe local molecular distributions at soft-hard interfaces in order to understand nanoscale variations in the growth and reactivity of colloidal nanocrystals.

We focus our analysis on two systems: CTAB-coated AuNRs, which are important for applications in plasmonics, diagnostics, and therapeutics, and serve as a model system for understanding structural and functional anisotropy in colloidal nanostructures [3], and MTAB-coated mini-AuNRs, which have applications in nanomedicine due to their biocompatibility and efficient cellular uptake [4]. We spraydeposit our nanoparticles onto graphene supports and use EELS spectrum imaging to map the molecular shell. The advantage of using a graphene support is twofold: it provides an ultra-low background that maximizes contrast of low-Z elements [5], and it has been shown to increase dose resistance as a result of its high thermal and electrical conductivity [6]. Figure 1 shows an annular-dark field (ADF) STEM image of two gold nanoparticles with the corresponding carbon and nitrogen elemental maps. These EELS maps show the CTAB $\left(\mathrm{C}_{19} \mathrm{H}_{42} \mathrm{BrN}\right)$ shell surrounding the rods (Figure $\left.1 \mathrm{~b}, \mathrm{c}\right)$. We used quantitative EELS of the $\mathrm{C}-\mathrm{K}$ edge to quantify the molecular distributions of CTAB and MTAB, using the graphene support as a calibration. Our maps show that the while the MTAB distribution is fairly uniform, the CTAB distribution is highly anisotropic, with on average a 50\% drop in carbon density at the poles of the particle.

Using our EELS maps, we calculated the local binding density for each particle to produce a population average distribution of surface molecules. Figure 2 a shows the resulting CTAB binding density profiles for 5 nanorods for each quarter of the particle (dots) along with the mean value for each position (black line). Because the nanorods are nominally symmetric across their vertical and horizontal axes, we plot all the unique data on the right half of the graph (positive position values); we then mirror the data across $\mathrm{x}=0$ as a guide to the eye. The data show that the CTAB binding density decreases to a minimum at the poles of the nanorods, where it is roughly half of the mean value $(0.50+/-0.25)$. We applied the same experimental process and analysis to MTAB-coated mini gold nanorods (Figure 2b) and observe no 
decrease in binding density at the ends of the rod. Our data directly confirms the long-standing hypothesis that there is a decrease in CTAB binding density at the ends of the nanorod.

\section{References:}

[1] A Smith et al., Analyst 142 (2017), p. 11.

[2] N Burrows et al., Journal of Physical Chemistry Letters 7 (2016), p. 632.

[3] S Lohse and CJ Murphy, Journal of the American Chemical Society 134, (2012), p. 15607.

[4] L Vigderman et al., Angewandte Chemie International Edition 51 (2011), p. 636.

[5] Z Lee et al., Nano Letters 9 (2009), p. 3365.

[6] G Algara-Siller et al., Applied Physics Letters 103 (2013), p. 203107.

[7] This work was supported by the Packard Foundation, NSF-MRSEC under Award Number DMR1720633, and NSF grants 1608743 and 1503408. The experimental work was carried out in part in the Materials Research Laboratory and made use of the Cornell Center for Materials Research Shared Facilities, which are supported through the NSF-MRSEC program (DMR-1719875).
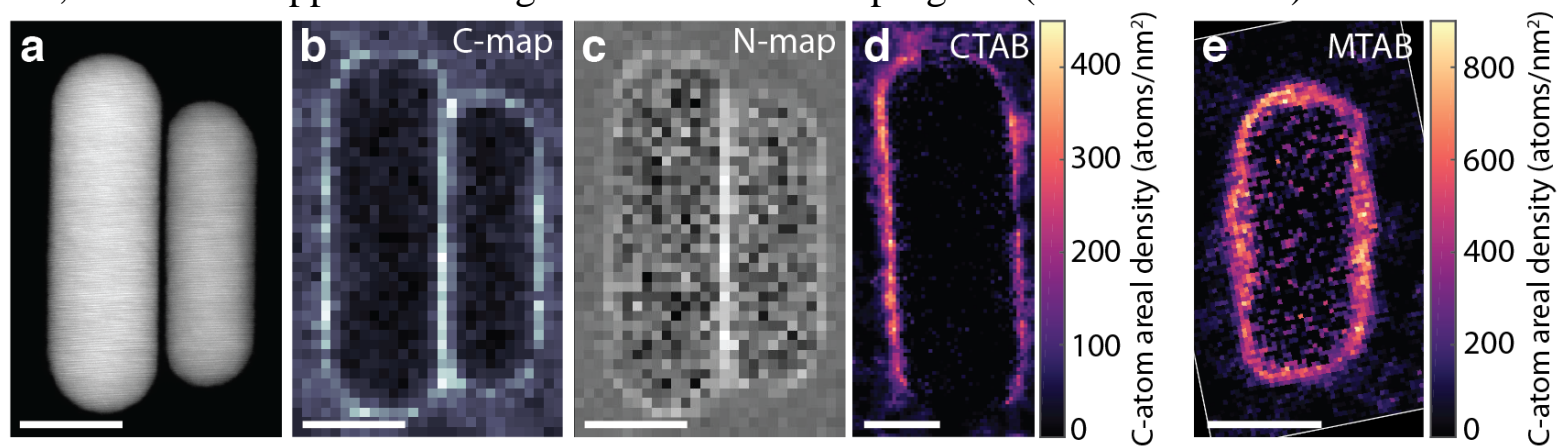

Figure 1. ADF-STEM image and EELS spectral maps of organic ligand-coated AuNRs. (a) ADF-STEM image of two CTAB-coated AuNRs deposited on a graphene support. (b, c) EELS spectral map of carbon (b) and nitrogen (c) from the same region as (a). CTAB contains 1 nitrogen per molecule, a $\mathrm{C}: \mathrm{N}$ ratio of 19:1, resulting in the lowered signal-to-noise in the nitrogen map. Scale bars are $15 \mathrm{~nm}$. Quantitative maps of the C-K edge of a representative (d) CTAB-coated AuNR and (e) MTAB-coated mini-AuNR with the graphene background subtracted. We rotated the MTAB map so that the particle is vertical; experimental data is within the white boundaries. Scale bars are $10 \mathrm{~nm}$.
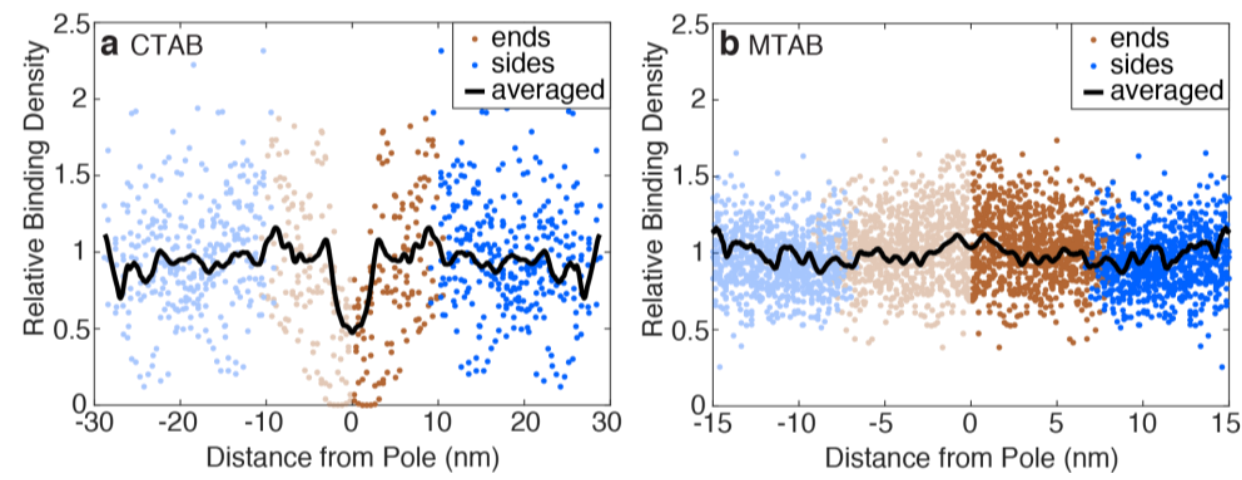

Figure 2. Variations in CTAB and MTAB distributions between multiple AuNRs. (a) CTAB-coated AuNR and (b) MTAB-coated mini-AuNR plots of the relative binding density as a function of position along the boundary, including data for individual rod quarters (scatter plot) and smoothed mean value (line plot). To compare positional trends in binding density, binding densities are normalized to the mean of each nanorod. All unique data is plotted for positive $\mathrm{x}$ values, and the plot is mirrored across $\mathrm{x}=0$. The $\mathrm{x}$-axes in (b) and (d) differ due to the difference in size between CTAB-coated AuNRs and MTAB-coated mini-AuNRs. 